\title{
New developments in CSF biomarkers for early detection and monitoring of Parkinson disease
}

$\mathrm{N}$ o biomarkers are currently available that can reliably diagnose Parkinson disease (PD) particularly in its early stages-or monitor the progression of this disorder. Two recently published reports have detailed progress in the use of cerebrospinal fluid (CSF) markers to achieve these goals.

In the first new study, Shi et al. explored the capability of a-synuclein, DJ-1 and five other proteins to distinguish PD from other parkinsonian conditions, and to monitor PD progression.

Previously, these researchers showed that among patients with PD or Alzheimer disease (AD) and healthy controls, low CSF levels of $\alpha$-synuclein and DJ-1-proteins implicated in PD pathogenesis-detected PD with high sensitivity but modest specificity. They also showed that changes in a panel of CSF markers including amyloid- $\beta_{1-42}\left(A \beta_{1-42}\right)$ and tau-which are often used as markers of $\mathrm{AD}$ - could strongly differentiate cases of PD from healthy and $\mathrm{AD}$ controls.

In the new study, the five additional proteins examined were $A \beta_{1-42}$, total and phosphorylated tau, and two markers of inflammation, Flt3 ligand and fractalkine. In total, the investigators measured the CSF levels of these proteins plus a-synuclein and DJ-1 in 137 healthy controls, 126 cases of PD, 50 cases of AD and 32 cases of multiple system atrophy (MSA). These data were then validated in independent cohorts of patients with PD.

"In the panel of markers evaluated, nothing was significantly better than $\alpha$-synuclein or DJ-1 alone in differentiating cases of PD from healthy controls," says Jing Zhang, principal investigator of the study.

However, some of the panel's proteins proved useful in distinguishing PD from other diseases. "From CSF levels of Flt3 ligand, PD could be clearly differentiated from MSA, a disease that overlaps with PD clinically," highlights Zhang.

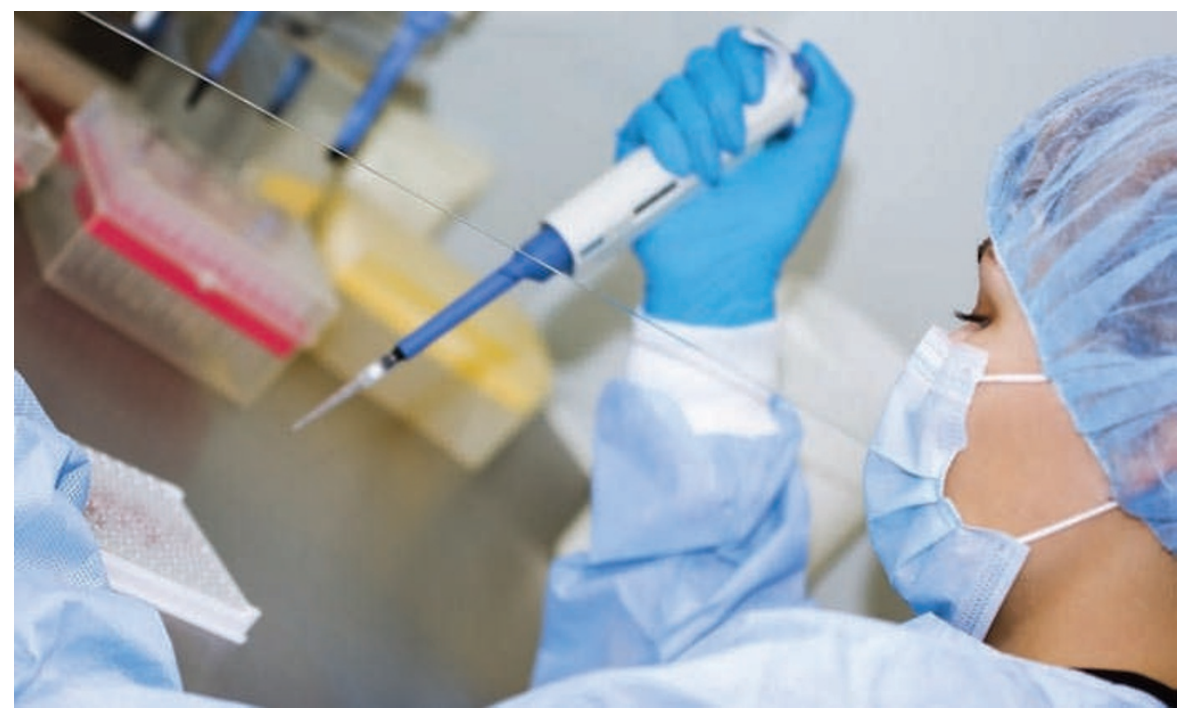

In addition to the panel's use in the differential diagnosis of PD, the researchers showed that the CSF fractalkine: $\mathrm{A} \beta_{1-42}$ ratio increased with rising PD severity.

"It looks like inflammation markers, as a class, are promising for PD differential diagnosis as well as monitoring progression," explains Zhang. "It also has become clear that $\mathrm{A} \beta$ and tau, typically used as markers for $\mathrm{AD}$ in the past, likely play major roles in PD as well."

In the second recently published study, Tokuda and colleagues evaluated soluble a-synuclein oligomers as potential early markers of PD. Emerging evidence suggests that these oligomeric species may have neurotoxic effects in this movement disorder.

Using enzyme-linked immunosorbent assays, the investigators measured both total and oligomeric $\alpha$-synuclein CSF levels in 32 patients with PD and 28 age-matched controls (16 neurologically normal individuals and 12 patients with various neurological diseases).

The results of these assays showed that both the level of CSF oligomeric a-synuclein and the oligomer:total a-synuclein ratio were increased in patients with PD compared with healthy and disease controls. Moreover, further analysis revealed that patients with early or mild PD showed the greatest increases in the levels of both markers over control values.

"If our results are confirmed in other cohorts, there is no doubt that $\alpha$-synuclein oligomers would be useful markers for early detection of PD," explains the study's principal investigator, Omar El-Agnaf. "It is very important to have a marker that could identify subjects at risk of developing PD, as this would offer the opportunity for early and effective treatment when drugs become available."

Looking to the future, both El-Agnaf and Zhang, and their groups, intend to validate their respective observations in larger studies.

Darran Yates

Original articles Shi, M. et al. Cerebrospinal fluid biomarkers for Parkinson disease diagnosis and progression. Ann. Neurol. doi:10.1002/ana.22311 | Tokuda, T. et al. Detection of elevated levels of $\alpha$-synuclein oligomers in CSF from patients with Parkinson disease. Neurology 75, 1766-1772 (2010) 